\title{
Middle Cranial Fossa Approach to Repair Tegmen Defects Assisted by 3-Dimensionally Printed Temporal Bone Models
}

Sameer Ahmed, MD ${ }^{+}$; Kyle K. VanKoevering, $\mathrm{MD}^{+}$; Stephanie Kline, MSE, MS ; Glenn E. Green, $\mathrm{MD}^{+} ; \mathrm{H}$. Alexander Arts, $\mathrm{MD}^{+}$

${ }^{+}$Department of Otolaryngology-Head and Neck Surgery, University of Michigan Health System * Section of Oral \& Maxillofacial Surgery, Department of Hospital Dentistry, University of Michigan Health System

Key words: Tegmen defect; skull base repair; 3-D printed temporal bone model; middle cranial fossa approach

Level of evidence (see Level of Evidence): Level 4 (case series)

Running title: Tegmen defect repair assisted by 3D models

Financial disclosures: None

Conflicts of interest: None currently but we have a patent pending for peripherally related work involving lateral skull base repair and 3D printed technology.

\section{Corresponding Author Information:}

Sameer Ahmed, M.D.

University of Michigan Health System

Department of Otolaryngology - Head and Neck Surgery

Division of Otology-Neurotology

1500 E. Medical Center Drive

1904 Taubman Center

Ann Arbor, MI 48109-5312

Phone: 734-936-8006

Fax: 734-936-9625

Email: sameahme@med.umich.edu

Abstract word count: 245 words

Manuscript word count (not including the abstract): 1975 words

This is the author manuscript accepted for publication and has undergone full peer review but has not been through the copyediting, typesetting, pagination and proofreading process, which may lead to differences between this version and the Version record. Please cite this article as doi:10.1002/ lary.26438. 


\section{$\underline{\text { Abstract }}$}

Objective: To explore the perioperative utility of three-dimensionally (3D) printed temporal bone models of patients undergoing repair of lateral skull base defects and spontaneous cerebrospinal fluid leaks with the middle cranial fossa approach.

Methods: Three dimensionally printed temporal bone models_- based on patient-specific, highresolution CT imaging - were constructed using inexpensive polymer materials. Pre-operatively, the models demonstrated the extent of temporal lobe retraction necessary to visualize the proposed defects in the lateral skull base. Also pre-operatively, silastic sheeting was arranged across the modeled tegmen, marked, and cut to cover all of the proposed defect sites. The silastic sheeting was then sterilized and subsequently served as a precise intraoperative template for a synthetic dural replacement graft. Of note, these grafts were customized without needing to retract the temporal lobe.

Results: Five patients underwent the middle cranial fossa approach assisted by 3D printed temporal bone models to repair tegmen defects and spontaneous CSF leaks. No complications were encountered. The prefabricated dural repair grafts were easily placed and fit precisely onto the middle fossa floor without any additional modifications. All defects were covered as predicted by the 3D temporal bone models. At their post-operative visits, all five patients maintained resolution of their spontaneous cerebrospinal fluid leaks.

Conclusion: Inexpensive 3D printed temporal bone models of tegmen defects can serve as beneficial adjuncts during lateral skull base repair. The models provide a panoramic preoperative view of all tegmen defects and allow for custom templating of dural grafts without temporal lobe retraction. 


\section{Introduction}

Defects in the lateral skull base (tegmen mastoideum and tegmen tympani) can be associated with cerebrospinal fluid leakage and temporal encephalocele formation, which increase the risk of meningitis in addition to causing aural fullness, pulsatile tinnitus or conductive hearing loss. ${ }^{1-3}$ Defects in the tegmen may be caused by trauma, chronic otitis media, erosive neoplastic disease, or, most commonly, a spontaneous idiopathic processes. For patients with spontaneous defects, gradual erosion of the tegmen from pulsations of the dura in patients with high intracranial pressures or aberrant arachnoid granulation formations through the middle fossa or posterior fossa plates are thought to be the underlying mechanisms. ${ }^{4,5}$ The role of obesity in the development of spontaneous cerebrospinal fluid otorrhea has been more closely examined, as Kutz et al found that this condition is more common in obese female patients. ${ }^{6}$ Stevens et al identified a statistically significant radiographic association of tegmen dehiscence in obese patients. ${ }^{7}$ Perhaps not coincidentally, the incidence of spontaneous cerebrospinal fluid leaks, temporal encephaloceles, and tegmen dehiscences appears to be increasing with the obesity epidemic. ${ }^{8}$

Tegmen defects can be repaired via one of three approaches: transmastoid, middle cranial fossa, or a combined transmastoid-middle fossa approach. ${ }^{9}$ The transmastoid approach avoids a craniotomy and provides sufficient exposure for postero-lateral tegmen mastoideum defects. However, for larger defects or those extending to the tegmen tympani or exposing the ossicular heads, the transmastoid approach fails to provide adequate exposure. The middle cranial fossa approach provides access to the entire lateral skull base, including regions medial to the ossicular heads or anteriorly near the petrous apex. The main disadvantage of the middle fossa approach is that it requires a craniotomy, which increases the risk of dural tears, subdural or epidural 
hematoma formation, meningitis, and temporal lobe injury. ${ }^{9,10}$ Many factors are important when selecting the approach, including age, comorbidities, history of prior repairs, and pre-existing hearing loss. Both autologous and alloplastic graft materials have been successfully used to repair lateral skull base defects. However, sculpting these grafts to fit a patient's specific skull base anatomy remains a challenging and often time-consuming part of the repair. ${ }^{6,11-14}$

We hypothesized that 3D printing could be utilized to improve the preoperative planning and expedite the fashioning of the grafts placed intraoperatively. Three-dimensional printing has allowed for rapid, cost-effective fabrication of patient-specific, highly complex models in various fields. ${ }^{15,16}$ Temporal bone models have been previously created with $3 \mathrm{D}$ printing technology primarily as simulation models to enhance resident education. ${ }^{17,18}$ However, to date, there are no reports of the 3D printed patient-specific temporal bone models to improve preoperative preparation and surgical repair. We describe the use of low-cost 3D printed temporal bone models to be used as templates to pre-operatively customize synthetic dural replacement grafts and intra-operatively verify that all tegmen defects and near-dehiscent sites have been addressed.

\section{Materials and Methods}

A retrospective review was performed of patients who underwent the middle cranial fossa approach to repair tegmen defects assisted by $3 \mathrm{D}$ printed temporal bone models. The Institutional Review Board at the University of Michigan approved this study (HUM00108678). All surgeries were performed by the senior surgeon (HAA) at a single tertiary care institution.

\section{Preparation of 3-D Printed Temporal Bone Models}


Pre-operatively, all patients underwent high-resolution CT imaging (0.625 mm slices). The digital imaging and communication (DICOM) images from each CT scan were then uploaded into a 3D modeling and segmentation program (Mimics, Materialise TM, Leuven, Belgium). The DICOM images were segmented using the thresholding function isolating the Hounsfield units consistent with the temporal bone. A 3D model of each patient's temporal bone was then generated (Figure 1). The temporal bones for each patient were then $3 \mathrm{D}$ printed on a fused deposition modeling (FDM) printer at a layer thickness of 100 micrometers using polylactic acid (PLA) or acrylonitrile butadiene styrene (ABS) [Figure 2]. These materials are inexpensive and readily available with most consumer and commercial grade FDM printers.

\section{Preparation of Synthetic Dural Grafts}

The 3-D printed temporal bone model displayed all of the frankly dehiscent sites and nearly dehiscent sites in the tegmen. This tangible, panoramic view of the lateral skull base provided the surgeon an understanding of the extent of craniotomy and temporal lobe retraction required in order to visualize all of the tegmen defects. A thin, flexible piece of silastic sheeting ( $0.5 \mathrm{~mm}$ thickness) was then arranged across the modeled tegmen, marked, and cut to cover all of the proposed defect sites (Figure 3). A notch was placed at the location of the middle meningeal artery. The shape of the silastic sheet was highly irregular and variable from patient to patient. The silastic sheet was then sterilized. Intra-operatively, the sterile silastic sheet served as a template for creating the appropriately sized synthetic dural replacement graft (DuRepair, Medtronic) which was cut to match the silastic template. The 3D printed model also served as a non-sterile guide during the procedure for surgical reference. 


\section{Surgical Technique}

A traditional temporal scalp flap was fashioned and the skin and temporalis were reflected inferiorly. A middle fossa craniotomy was performed and the temporal lobe was gently retracted. A Greenberg retractor was utilized to facilitate exposure of lateral skull base defects (Figure 4). The non-sterile model was then re-inspected to ensure all tegmen defects were visualized in-situ. Calvarial bone grafts were harvested from the bone flap and fashioned to span the largest defects. Once adequate exposure was obtained, the bone grafts were then secured with carbonated apatite bone cement (CraniOs, Synthes). The previously customized synthetic dural replacement graft was then placed into anatomic position along the floor of the middle cranial fossa to provide a water-tight seal (Figure 5). Tissue glue was placed and the Greenberg retractor was relaxed as the temporal lobe was allowed to passively return. The craniotomy bone flap was replaced and the wound closed in a layered fashion. For some patients who had multiple small tegmen defects, the synthetic dural replacement graft was used in isolation.

\section{Results}

Five patients underwent the middle cranial fossa approach to repair tegmen defects and spontaneous CSF leaks assisted by 3D printed temporal bone models. Three-dimensionally printed temporal bone models were manufactured for each of these five patients. The resulting models compared favorably to the tegmen anatomy based upon imaging and intraoperative findings. Raw material cost was less than $\$ 5$ per PLA model, $\$ 5$ per ABS model, and $\$ 7$ per silastic sheet.

Three patients were female and two patients were male. All five patients presented with CSF otorrhea after tympanostomy tube placement performed at outside institutions. The 
tympanostomy tubes were all removed pre-operatively and the tympanic membranes had healed spontaneously. One patient had a dehiscence of the superior semicircular canal, which was repaired along with his tegmen defects (Patient \#2). Patient characteristics and surgical outcomes are listed in Table 1.

All five patients underwent successful repair of their tegmen defects without complications. The prefabricated dural repair grafts were easily placed and fit precisely onto the middle fossa floor without any additional modifications. All defects were covered as predicted by the $3 \mathrm{D}$ models. At their post-operative visits, all patients maintained resolution of their spontaneous cerebrospinal fluid leaks, which was verified by the use of binocular otomicroscopy and formal tympanometry.

\section{Discussion}

Reconstruction of defects in the middle fossa floor can be a challenging and frustrating procedure, and recurrence rates have been described as high as $28 \% .^{9}$ One of the main challenges lies in obtaining adequate exposure to visualize all tegmen defects, especially anteromedial defects that lie near the geniculate ganglion. Use of 3-D printed temporal bone models aids in the visualization of all the tegmen defects and near-dehiscent sites preoperatively by providing a panoramic view that translates well to the view obtained in the operating room. Such models can be made from inexpensive materials and maintain high fidelity in demonstrating the tegmen anatomy. The 3D model of the lateral skull base provides the surgeon with a tangible display of all defects needing to be addressed, which improves surgical confidence that adequate exposure has been obtained. 
Notably, while it is difficult to quantify this operative finding, the tegmen defects appeared to be slightly overestimated by the CT scan (and the subsequent CT-based 3D models). This is likely due to signal averaging of near-dehiscent regions. However, we strongly believe that repair of these near-dehiscent regions in addition to frankly dehiscent sites is critical to a durable repair and avoiding delayed failures.

Pre-fabricating a template for lateral skull base repair is a distinct advantage of the preoperative 3D temporal bone models. Silastic sheeting can be cut and fashioned to exactly match the compromised lateral skull base of the models, and can be subsequently sterilized and utilized intraoperatively. This helps reduce the degree of temporal lobe retraction, as the dural graft does not need to be directly sized to the tegmen intraoperatively. Once adequate exposure of the lateral skull base is obtained, the synthetic dural graft can be placed quickly and with a high level of confidence knowing that all defects and nearly-dehiscent sites are covered.

Currently, inexpensive materials available for 3D printing are difficult to sterilize due to their thermal properties. Our group is exploring options for materials that can be $3 \mathrm{D}$ printed with high fidelity and low cost and that can be reliably autoclaved. These materials should help to streamline the process of lateral skull base repair with 3D printed models.

Our study has certain limitations. We believe that these repairs can be completed more quickly — with a shorter duration of brain retraction — with the use of 3D printed temporal bone models, because the graft materials used for repair are customized before temporal lobe retraction occurs. However, we did not meticulously time the pre-fabricated repair in comparison to that of historical controls. In retrospect, the senior surgeon (H.A.A.) noticed a significant difference in time and ease of graft placement; however, we do not currently have timed data to validate this notion. Secondly, tegmen defects tend to be overestimated in the $3 \mathrm{D}$ 
models. This can lead to some uncertainty about whether a defect in the model is a true dehiscence or near-dehiscence. Our approach has been to cover all modeled defects, even if they ultimately represent a clinical near-dehiscence. We believe this may be beneficial in establishing a durable treatment benefit, as near-dehiscent sites may develop into frank dehiscence in the future. Notably, the silastic sheet template in Figure 3 covers the frankly dehiscent and neardehiscent sites, while avoiding the skull base foramina and groove for the middle meningeal artery and its branches along the inner surface of the lateral skull. Thirdly, our follow-up was short, ranging from four to seven months. Since we are reporting initial results on a novel technique, we do not yet have long-term data to validate a durable resolution of CSF leakage. As we continue to follow patients over time, we hope to obtain long-term data that validates this hypothesis.

A final concern is the accessibility of this technology to clinicians. In recent years, access to $3 \mathrm{D}$ printing technology has grown exponentially as multiple applications of this technology have been well described. ${ }^{19}$ With improvements in low cost machines, high resolution desktop printers can now be purchased for around $\$ 1,000$. The particular setup used in this modeling process cost $\$ 1,200$. As printers become more affordable, we anticipate this technology will be accessible at most academic medical centers in the next two to three years either through the hospital or engineering departments. Furthermore, modeling software continues to improve, with many free software programs utilizing near-automated segmentation processes to simplify 3D modeling.

\section{Conclusion}

Three-dimensionally printed temporal bone models of tegmen defects can serve as beneficial adjuncts during lateral skull base repair. Such models are inexpensive and can be 
made with most consumer or commercially available 3-D printers. The models provide a panoramic pre-operative view of all tegmen defects, increase surgeon confidence during and after the repair, and allow for preoperative custom templating of dural grafts without temporal lobe retraction.
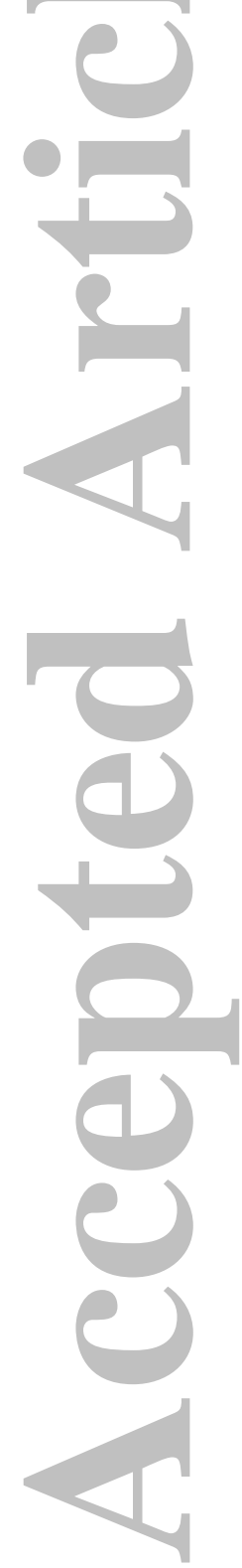

John Wiley \& Sons

This article is protected by copyright. All rights reserved. 
Acknowledgements: None
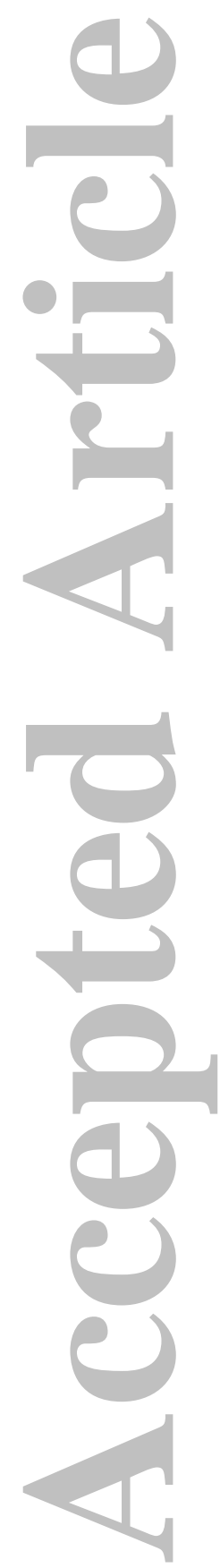

John Wiley \& Sons

This article is protected by copyright. All rights reserved. 


\section{References}

1. Brown NE, Grundfast KM, Jabre A, Megerian CA, O'Malley BW Jr, Rosenberg SI. Diagnosis and management of spontaneous cerebrospinal fluid-middle ear effusion and otorrhea.

Laryngoscope, 2004. 114(5): p. 800-5.

2. Sanna M, Fois P, Russo A, Falcioni M. Management of meningoencephalic herniation of the temporal bone: Personal experience and literature review. Laryngoscope, 2009. 119(8): p. 157985.

3. Savva A, Taylor MJ, Beatty CW. Management of cerebrospinal fluid leaks involving the temporal bone: report on 92 patients. Laryngoscope, 2003. 113(1): p. 50-6.

4. Gacek RR, Gacek MR, Tart R. Adult spontaneous cerebrospinal fluid otorrhea: diagnosis and management. Am J Otol, 1999. 20(6): p. 770-6.

5. Kaufman B, Yonas H, White RJ, Miller CF 2nd. Acquired middle cranial fossa fistulas: normal pressure and nontraumatic in origin. Neurosurgery, 1979. 5(4): p. 466-72.

6. Kutz JW Jr, Husain IA, Isaacson B, Roland PS. Management of spontaneous cerebrospinal fluid otorrhea. Laryngoscope, 2008. 118(12): p. 2195-9.

7. Stevens SM, Lambert PR, Rizk H, McIlwain WR, Nguyen SA, Meyer TA. Novel radiographic measurement algorithm demonstrating a link between obesity and lateral skull base attenuation. Otolaryngol Head Neck Surg, 2015. 152(1): p. 172-9.

8. Nelson RF, Gantz BJ, Hansen MR. The rising incidence of spontaneous cerebrospinal fluid leaks in the United States and the association with obesity and obstructive sleep apnea. Otol Neurotol, 2015. 36(3): p. 476-80. 
9. Gonen L, Handzel O, Shimony N, Fliss DM, Margalit N. Surgical management of spontaneous cerebrospinal fluid leakage through temporal bone defects-case series and review of the literature. Neurosurg Rev, 2016. 39(1): p. 141-50.

10. Betka J, Zverina E, Balogova Z, et al. Complications of microsurgery of vestibular schwannoma. Biomed Res Int, 2014. 2014: p. 315952.

11. Kveton JF, Coelho DH. Hydroxyapatite cement in temporal bone surgery: a 10 year experience. Laryngoscope, 2004. 114(1): p. 33-7.

12. Moffat DA, da Cruz MJ, Batten A, Hardy DG. Use of autologous osteocyte containing bone pate for closure of tegmental defects. Am J Otol, 1998. 19(6): p. 819-23.

13. Jahrsdoerfer RA, Richtsmeier WJ, Cantrell RW. Spontaneous CSF otorrhea. Arch Otolaryngol, 1981. 107(4): p. 257-61.

14. Gubbels SP, Selden NR, Delashaw JB Jr, McMenomey SO. Spontaneous middle fossa encephalocele and cerebrospinal fluid leakage: diagnosis and management. Otol Neurotol, 2007. 28(8): p. 1131-9.

15. Murphy SV, Atala A. 3D bioprinting of tissues and organs. Nat Biotechnol, 2014. 32(8): p. $773-85$.

16. Zopf DA, Hollister SJ, Nelson ME, Ohye RG, Green GE. Bioresorbable airway splint created with a three-dimensional printer. N Engl J Med, 2013. 368(21): p. 2043-5.

17. Mowry SE, Jammal H, Myer C 4th, Solares CA, Weinberger P. A Novel Temporal Bone Simulation Model Using 3D Printing Techniques. Otol Neurotol, 2015. 36(9): p. 1562-5. 18. Longfield EA, Brickman TM, Jeyakumar A. 3D Printed Pediatric Temporal Bone: A Novel Training Model. Otol Neurotol, 2015. 36(5): p. 793-5. 
19. VanKoevering KK, Hollister SJ, Green GE. Advances in 3-Dimensional Printing in Otolaryngology: A Review. JAMA Otolaryngol Head Neck Surg. 2016 Oct 6.

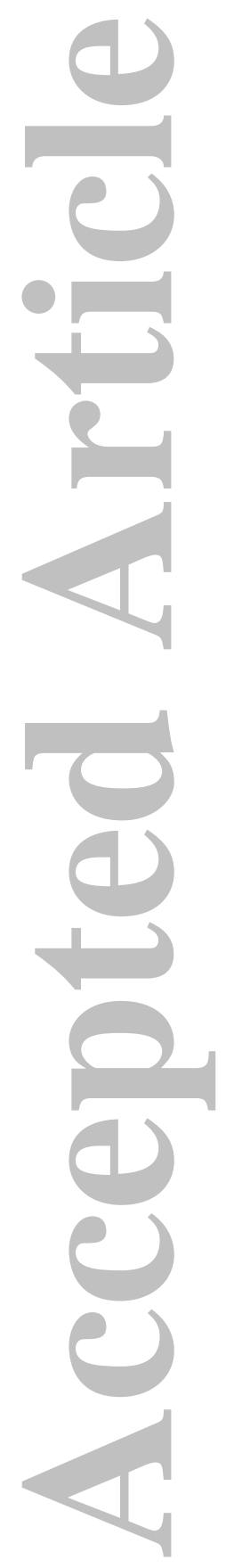

John Wiley \& Sons

This article is protected by copyright. All rights reserved. 


\section{Figure and Table Legends:}

Figure 1: Computer aided design of a temporal bone model with multiple tegmen mastoideum and tegmen tympani defects in the left lateral skull base for Patient \#2. Low power view (A) and high power view (B).

Figure 2: (A) Low-power view of a three dimensionally printed temporal bone model for Patient \#2. (B) High power view of the same model demonstrating multiple tegmen mastoideum and tegmen tympani defects are visible along with exposure of the ossicle heads $(*)$ and dehiscence of the superior semicircular canal (oval).

Figure 3: Silastic sheeting customized in order to cover dehiscent and nearly dehiscent sites in the tegmen for Patient \#2. The clear silastic sheeting is outlined. Note that foramen spinosum is not covered by the silastic.

Figure 4: Intra-operative view of multiple tegmen defects and nearly dehiscent sites in the right lateral skull base for Patient \#5.

Figure 5: The customized synthetic dural graft placed along the floor of the middle cranial fossa for Patient \#5.

Table 1: Patient characteristics 
Table 1: Patient characteristics

\begin{tabular}{|l|l|l|l|l|l|l|}
\hline $\begin{array}{c}\text { Patient } \\
\#\end{array}$ & Gender & $\begin{array}{c}\text { Age } \\
\text { (years) }\end{array}$ & $\begin{array}{c}\text { BMI } \\
\left(\mathbf{k g} / \mathbf{m}^{2}\right)\end{array}$ & $\begin{array}{c}\text { Location of Tegmen } \\
\text { Defects }\end{array}$ & $\begin{array}{l}\text { Length of } \\
\text { follow-up }\end{array}$ & $\begin{array}{c}\text { CSF leak } \\
\text { repaired? }\end{array}$ \\
\hline 1 & F & 61 & 34 & $\begin{array}{l}\text { Multiple small defects } \\
\text { of tegmen mastoideum } \\
\text { and tegmen tympani }\end{array}$ & 4 months & Yes \\
\hline 2 & M & 63 & 27 & $\begin{array}{l}\text { Large defect in the } \\
\text { tegmen mastoideum } \\
\text { and large defect in the } \\
\text { tegmen tympani }\end{array}$ & 7 months & Yes \\
\hline 3 & F & 55 & 34 & $\begin{array}{l}\text { One small defect of the } \\
\text { tegmen mastoideum } \\
\text { and two small defects } \\
\text { in the tegmen tympani }\end{array}$ & 4 months & Yes \\
\hline 4 & F & 48 & 29 & $\begin{array}{l}\text { Multiple small defects } \\
\text { of the tegmen } \\
\text { mastoideum and one } \\
\text { large defect overlying } \\
\text { the external auditory } \\
\text { canal }\end{array}$ & 4 months & Yes \\
\hline 5 & M & 41 & 30 & $\begin{array}{l}\text { Multiple small defects } \\
\text { of the tegmen } \\
\text { mastoideum and one } \\
\text { large defect of the } \\
\text { tegmen tympani }\end{array}$ & 6 months & Yes \\
\hline
\end{tabular}





Figure 1: Computer aided design of a temporal bone model with multiple tegmen mastoideum and tegmen tympani defects in the left lateral skull base for Patient \#2. Low power view (A) and high power view (B).



$243 \times 119 \mathrm{~mm}(150 \times 150 \mathrm{DPI})$ 

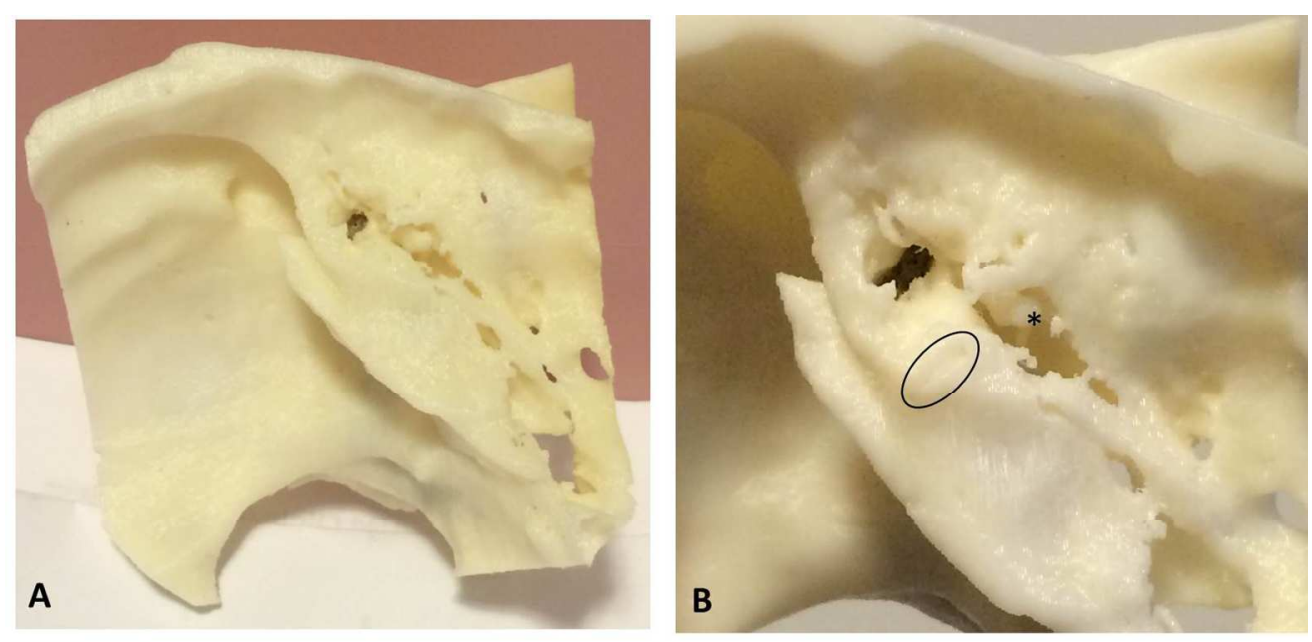

Figure 2: (A) Low-power view of a three dimensionally printed temporal bone model for Patient \#2. (B) High power view of the same model demonstrating multiple tegmen mastoideum and tegmen tympani defects are visible along with exposure of the ossicle heads $(*)$ and dehiscence of the superior semicircular canal (oval).

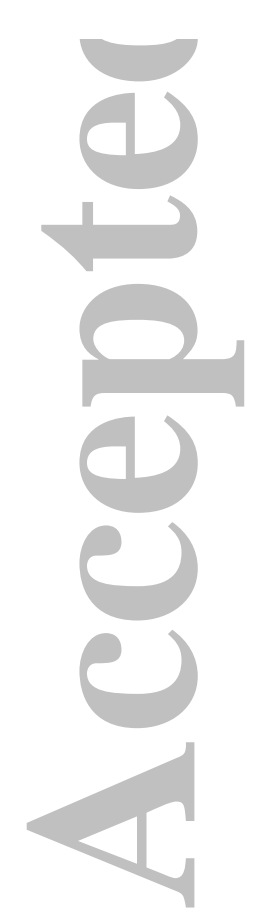

$288 \times 139 \mathrm{~mm}(150 \times 150 \mathrm{DPI})$ 


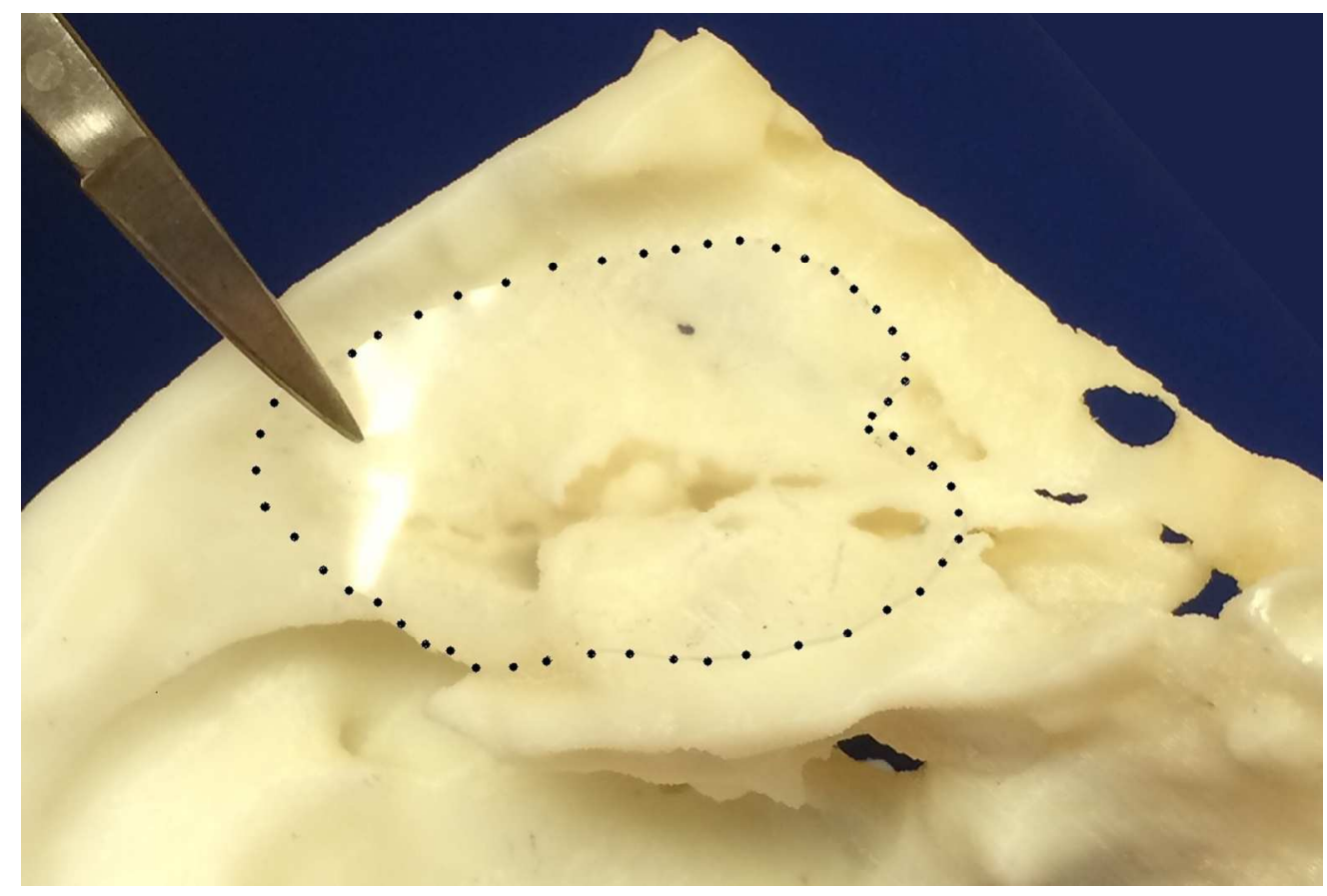

Figure 3: Silastic sheeting customized in order to cover dehiscent and nearly dehiscent sites in the tegmen for Patient \#2. The clear silastic sheeting is outlined.

$322 \times 216 \mathrm{~mm}(96 \times 96$ DPI $)$

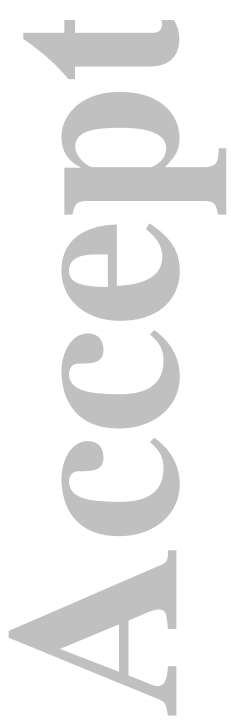




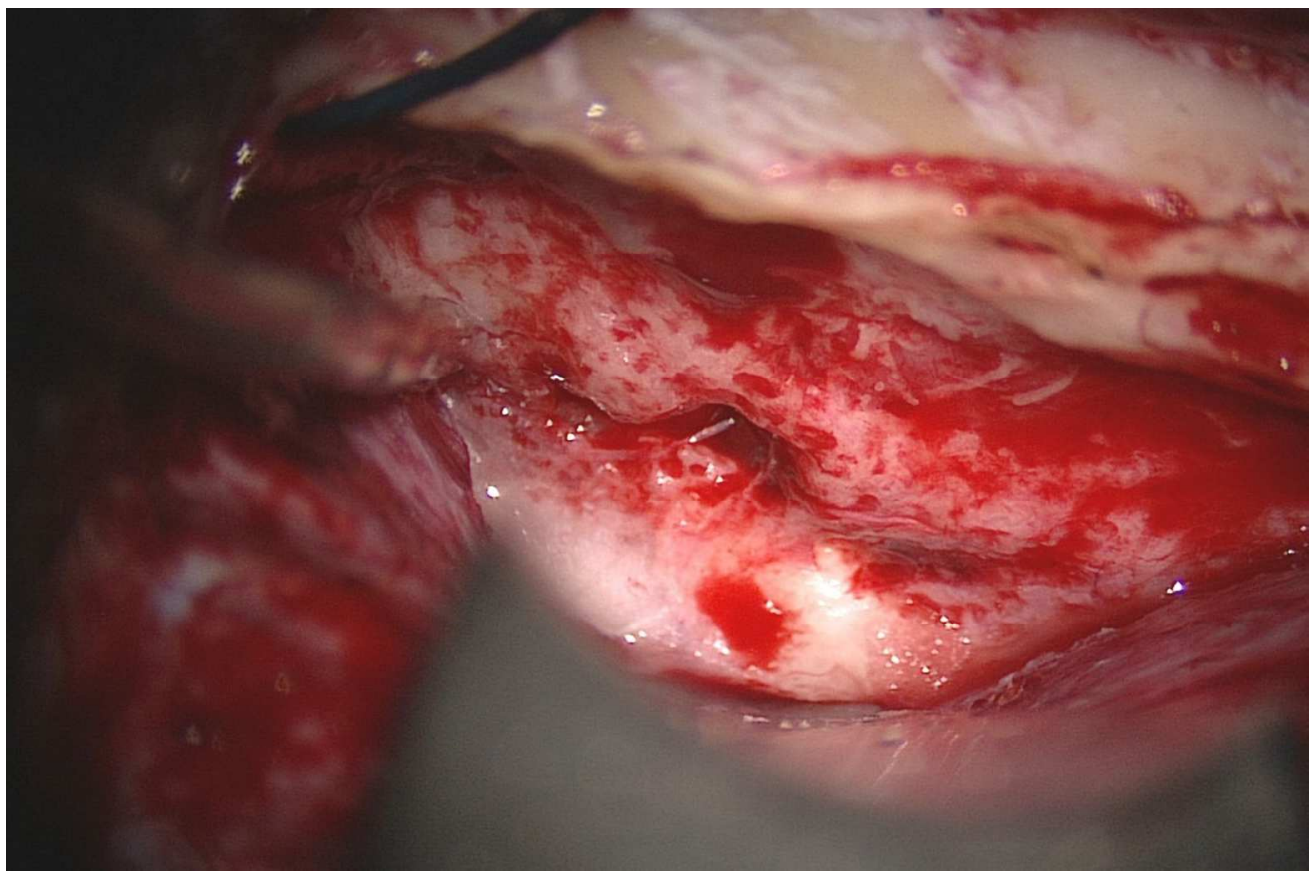

Figure 4: Intra-operative view of multiple tegmen defects and nearly dehiscent sites in the right lateral skull base for Patient \#5.

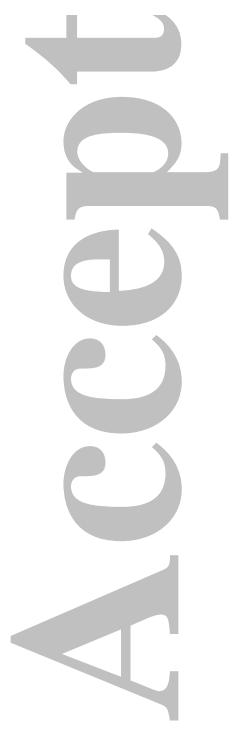

$395 \times 261 \mathrm{~mm}(96 \times 96 \mathrm{DPI})$ 


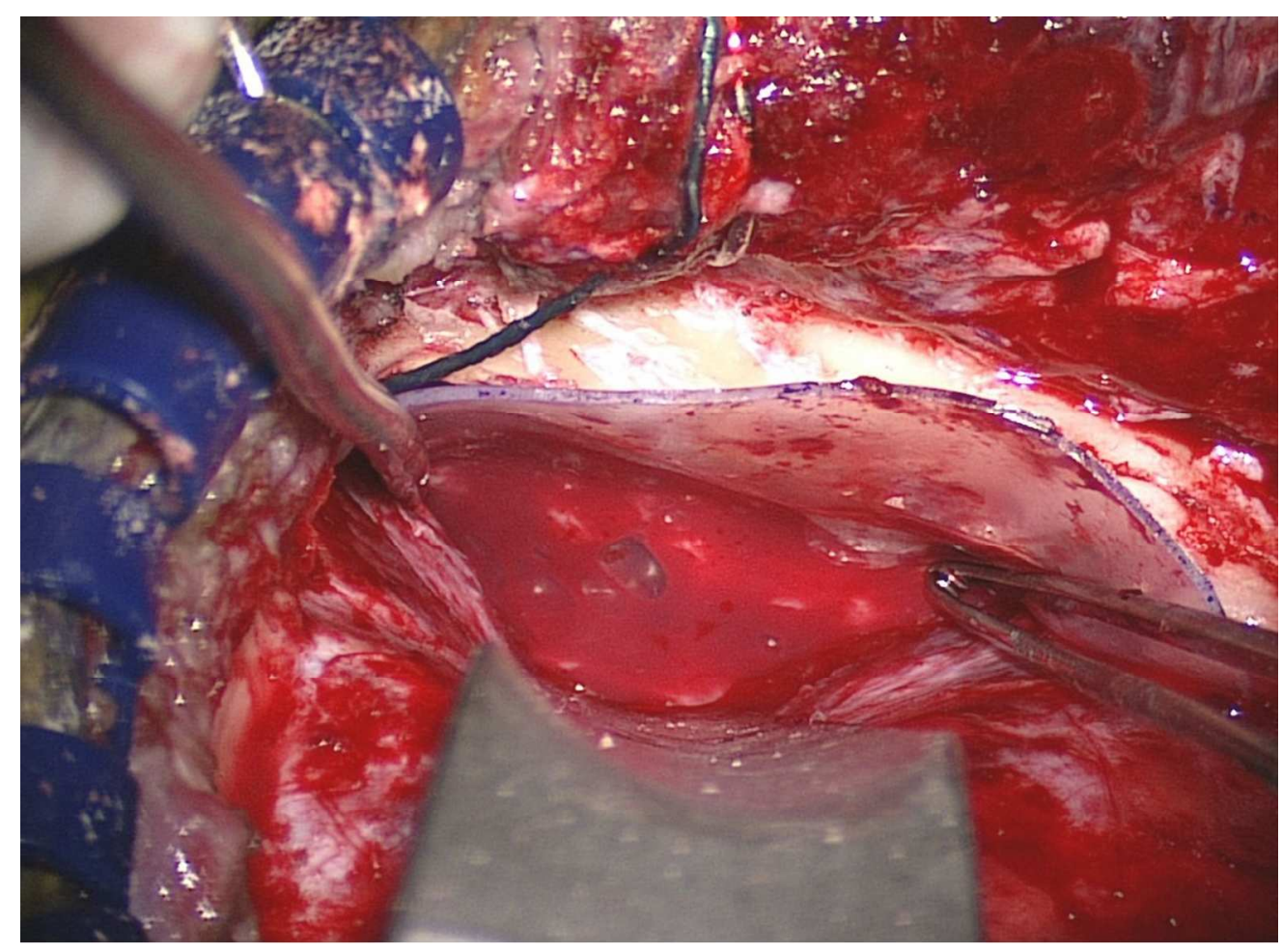

Figure 5: The customized synthetic dural graft placed along the floor of the middle cranial fossa for Patient \#5.

$330 \times 243 \mathrm{~mm}(96 \times 96$ DPI $)$

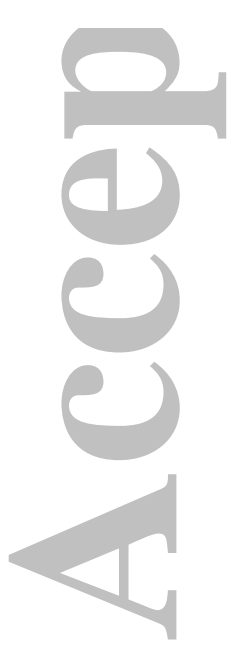

John Wiley \& Sons

This article is protected by copyright. All rights reserved. 